\title{
Research of Transfer Alignment for Airborne Distributed Inertial Attitude Measurement System
}

\author{
Yukun Wang, Shuai Chen, Xian Li, Runwu Zhong \\ Department of Automation \\ Nanjing University of Science and Technology \\ Nanjing, China \\ e-mail:wyk521215@163.com
}

\begin{abstract}
According to airborne early warning system's higher and higher requirement for precise attitude information and increasing demand for local attitude reference, a distributed inertial attitude measurement system scheme which is based on the information of airborne MINS (master inertial navigation system) and the information of several short precision SINS (slave inertial navigation system) was designed. The ground-demo of the distributed inertial attitude measurement system was set up to test the proposed method. Then to reduce the effect of the deflection to transfer alignment, the integral velocity and the integral angular rate are broadened to the state variables of Kalman filter. Besides, the state equation and the measurement equation are derived in detail. The simulation results show that the precisions of horizontal angle and azimuth angle are superior to $1.44^{\prime}$ and $0.48^{\prime}$ respectively. All these characteristics show that the scheme is feasible and can provide reference for the engineering application of the distributed inertial attitude measurement system.
\end{abstract}

Keywords-distributed inertial attitude measurement system; transfer alignment; integral velocity plus integral angular rate; Kalman filter

\section{INTRODUCTION}

Facing the rapidly changing air situation, the pilot must find the target in the most agile manner, and choose the most effective way to attack. In order to enhance the pilot's observation range of the vision field to the outside, six optical sensors for imaging are installed in the aircraft and eventually a variety of information within the range of $360^{\circ}$ is able to be collected[1]. However, line-of-sight for optical imaging detector with the carrier aircraft vibration must produce a certain level of random jitter. Such a vibration will make the image generation shaking, and will have a significant impact on the image mosaic of several detectors. Therefore it is necessary to install a specialized SINS used to measure the position of the sensor in each of the optical sensor. However, if each sensor location is installed a highprecision SINS, it will increase the costs.

Distributed inertial attitude measurement system relies on SINS transfer alignment to ensure accuracy. Transfer alignment uses MINS parameters such as speed, attitude and angular rate to match SINS parameters and then estimates SINS misalign angle[2-3].Finally, it can output platform attitude, position and velocity information in real-time.

\section{SYSTEM DESCRIPTION}

In this system, Kalman filter is used as the primary method of estimation. This is because, Kalman filter is optimal filter. Therefore, in the following studies, the transfer alignment algorithm is designed by using the Kalman filter that utilize the corresponding matching method. The filter model concludes the state equation and the measurement equation .

A. State equation of transfer alignment system

Attitude error equation[4-6]:

$$
\dot{\varphi}^{n}=-\omega_{i n}^{n} \times \varphi^{n}+\delta \omega_{i n}^{n}+C_{b}^{n} \varepsilon^{b}
$$

Velocity error equation:

$$
\begin{aligned}
\delta \dot{V}^{n} & =f^{n} \times \varphi^{n}-\left(2 \delta \omega_{i e}^{n}+\omega_{e n}^{n}\right) \times \delta V^{n} \\
& -\left(2 \omega_{i e}^{n}+\omega_{e n}^{n}\right) \times \delta V^{n}+C_{b}^{n} \nabla^{b}
\end{aligned}
$$

Inertial device error equation:

$$
\begin{aligned}
& \dot{\varepsilon}^{b}=0 \\
& \dot{\nabla}^{b}=0
\end{aligned} .
$$

Integral velocity error equation:

$$
I \dot{V}=\delta V^{n} .
$$

Integral angular rate error equation:

$$
I \dot{W}=\left(\omega_{i a}^{n} \times\right) \varphi+C_{b}^{n} \varepsilon^{b}+\eta
$$

Where $\varphi^{n}$ and $\delta V^{n}$ are the attitude error and velocity error vectors respectively; $V^{n}$ indicates velocity vector in navigation coordinate; $f^{n}$ indicates specific force in navigation coordinate; $\omega_{i e}^{n}$ is the earth rate vector; $\omega_{e n}^{n}$ is the turn rate of the navigation frame with respect to the Earth; 
$\varepsilon^{b}$ and $\nabla^{b}$ are equivalent gyro drift vector and equivalent accelerometer bias vector respectively in the carrier coordinate system.$C_{b}^{n}$ is a direction cosine matrix ; $I \dot{V}$ indicates differentiation of integral velocity ; $I \dot{W}$ indicates differentiation of integral angular rate; $\omega_{i a}^{n}$ is the rotation rate of MINS with respect to inertial frame defined in navigation frame; $\eta$ indicates white noise.

The mathematical model of the system state is:

$$
\dot{X}=F X+G W
$$

Where

$$
\begin{aligned}
& X=\left[\varphi^{n}, \delta V^{n}, \varepsilon^{b}, \nabla^{b}, I V, I W\right]^{\mathrm{T}} \\
& F=\left[\begin{array}{ccccc}
-\left[\omega_{i n}^{n} \times\right] & 0_{3 \times 3} & C_{b}^{n} & 0_{3 \times 3} & 0_{3 \times 6} \\
{\left[f^{n} \times\right]} & -\left[\left(2 \omega_{i e}^{n}+\omega_{e n}^{n}\right) \times\right] & 0_{3 \times 3} & C_{b}^{n} & 0_{3 \times 6} \\
0_{6 \times 3} & 0_{6 \times 3} & 0_{6 \times 3} & 0_{6 \times 3} & 0_{6 \times 6} \\
0_{3 \times 3} & I_{3 \times 3} & 0_{3 \times 3} & 0_{3 \times 3} & 0_{3 \times 6} \\
{\left[\omega_{i a}^{n} \times\right]} & 0_{3 \times 3} & C_{b}^{n} & 0_{3 \times 3} & 0_{3 \times 6}
\end{array}\right] \\
& G=\left[\begin{array}{cc}
C_{b}^{n} & 0_{3 \times 3} \\
0_{3 \times 3} & C_{b}^{n} \\
0_{12 \times 3} & 0_{12 \times 3}
\end{array}\right] \\
& W=\left[\begin{array}{llllll}
w_{\varepsilon x} & w_{\varepsilon y} & w_{\varepsilon z} & w_{\nabla x} & w_{\nabla y} & w_{\nabla z}
\end{array}\right]^{\mathrm{T}} .
\end{aligned}
$$

Where, $X$ is the state vector; $F$ is the state transition matrix; $G$ is the white noise driving matrix and $W$ is the white noise process vector.

\section{B. Transfer alignment measurement equation}

Integral velocity is defined as follows:

$$
I V=\int_{0}^{\Delta \mathrm{t}}\left(V_{s}^{n}-V_{m}^{n}\right) \mathrm{dt}
$$

Where, $\Delta \mathrm{t}$ indicates integral cycle; $V_{s}^{n}$ is velocity of SINS with respect to navigation frame; $V_{m}^{n}$ is velocity of MINS with respect to navigation frame .

Taking the derivative of (7), therefore:

$$
I \dot{V}=V_{s}^{n}-V_{m}^{n}
$$

Augmenting " $I V$ " as the new Kalman state, the observation equation for the integral of velocity can be represented as follows:

$$
Z_{v}^{o b s}=\int_{0}^{\Delta \mathrm{t}}\left(V_{s}^{n}-V_{m}^{n}\right) \mathrm{dt}
$$

The measurement equation for the integral of velocity can be represented as follows:

$$
Z_{v}=I V
$$

Integral angular rate is defined as follows:

$$
I W=\int_{0}^{\Delta \mathrm{t}} \omega_{i b}^{n} \mathrm{dt}-\int_{0}^{\Delta \mathrm{t}} \omega_{i a}^{n} \mathrm{dt}
$$

Where, $\Delta \mathrm{t}$ indicates integral cycle; $\omega_{i b}^{n}$ is the rotation rate of SINS with respect to inertial frame defined in navigation frame .

Taking the derivative of (11), therefore:

$$
I \dot{W}=\omega_{i b}^{n}-\omega_{i a}^{n}=\left(\omega_{i a}^{n} \times\right) \varphi+C_{b}^{n} \varepsilon^{b}+\eta .
$$

Augmenting " $I W$ " as the new Kalman state, the observation equation for the integral of angular rate can be represented as follows:

$$
Z_{w}^{o b s}=\int_{0}^{\Delta \mathrm{t}} \omega_{i b}^{n} \mathrm{dt}-\int_{0}^{\Delta \mathrm{t}} \omega_{i a}^{n} \mathrm{dt}
$$

The measurement equation for the integral of angular rate can be represented as follows:

$$
Z_{\omega}=I W
$$

The mathematical model of the measurement equation is :

$$
Z=H X+V
$$

Where

$$
\begin{gathered}
Z=\left[\begin{array}{ll}
Z_{v} & Z_{w}
\end{array}\right]^{\mathrm{T}} \\
H=\left[\begin{array}{lll}
0_{3 \times 12} & I_{3 \times 3} & 0_{3 \times 3} \\
0_{3 \times 12} & 0_{3 \times 3} & I_{3 \times 3}
\end{array}\right] .
\end{gathered}
$$




\section{Filter equations}

For discrete linear system, the state equation and the measurement equation are

$$
\left\{\begin{array}{l}
X_{k}=F_{k, k-1} X_{k-1}+\Gamma_{k-1} W_{k-1} \\
Z_{k}=H_{k} X_{k}+V
\end{array} .\right.
$$

Where $F_{k, k-1}$ is the system state transition matrix; $H_{k}$ is the measurement matrix; $W_{k-1}$ is the process noise vector; $V_{k}$ is the measurement noise vector. Both $V_{k}$ and $W_{k-1}$ are assumed to be uncorrelated zero mean Gaussian white noise sequence with covariance. The recursive formulas of Kalman filter are depicted as follows[7-8]:

$$
\left\{\begin{array}{l}
\hat{X}_{k, k-1}=F_{k, k-1} \hat{X}_{k-1} \\
\hat{X}_{k}=\hat{X}_{k, k-1}+K_{k}\left[Z_{k}-H_{k} \hat{X}_{k, k-1}\right] \\
K_{k}=P_{k, k-1} H_{k}^{T}\left[H_{k} P_{k, k-1} H_{k}^{T}+R_{k}\right]^{-1} \\
P_{k, k-1}=F_{k, k-1} P_{k-1} F_{k, k-1}^{T}+\Gamma_{k-1} Q_{k-1} \Gamma_{k-1}^{T} \\
P_{k}=\left[I-K_{k} H_{k}\right] P_{k, k-1}
\end{array} .\right.
$$

\section{RESULTS AND ANALYSIS}

In the following simulation, the performance of transfer alignment algorithm is evaluated under a typical trajectory which last for $100 \mathrm{sec}$ and consists of consecutive straight maneuvering and horizontal coordinated turn maneuvers. MEMS IMU statistics are as follows: gyro bias is $10^{\circ} / \mathrm{h}$; accelerometer bias is $5 \mathrm{mg}$. Initial values of Kalman filter are as follows:

$$
\begin{gathered}
X_{0}=0 \\
P_{0}(1,1)=P_{0}(2,2)=P_{0}(3,3)=\left(0.5^{\circ}\right)^{2} \\
P_{0}(4,4)=P_{0}(5,5)=P_{0}(6,6)=(0.1 \mathrm{~m} / \mathrm{s})^{2} \\
P_{0}(7,7)=P_{0}(8,8)=P_{0}(9,9)=\left(10^{\circ} / \mathrm{h}\right)^{2} \\
P_{0}(10,10)=P_{0}(11,11)=P_{0}(12,12)=(5 \mathrm{mg})^{2} \\
P_{0}(13,13)=P_{0}(14,14)=P_{0}(15,15)=(1)^{2} \\
P_{0}(16,16)=P_{0}(17,17)=P_{0}(18,18)=(0.0048)^{2} \\
Q_{0}(1,1)=Q_{0}(2,2)=Q_{0}(3,3)=\left(10^{\circ} / \mathrm{h}\right)^{2}
\end{gathered}
$$

$$
\begin{aligned}
& Q_{0}(4,4)=Q_{0}(5,5)=Q_{0}(6,6)=(5 \mathrm{mg})^{2} \\
& R_{0}(1,1)=R_{0}(2,2)=R_{0}(3,3)=(0.005)^{2} \\
& R_{0}(4,4)=R_{0}(5,5)=R_{0}(6,6)=(0.25)^{2}
\end{aligned}
$$

The transfer alignment scheme called integrated velocity and integrated angular rate matching is shown in Fig.1.System structure is shown in Fig.2.Pitch error estimation is shown in Fig.3;Roll error estimation is shown in Fig.4;Head error estimation is shown in Fig.5.

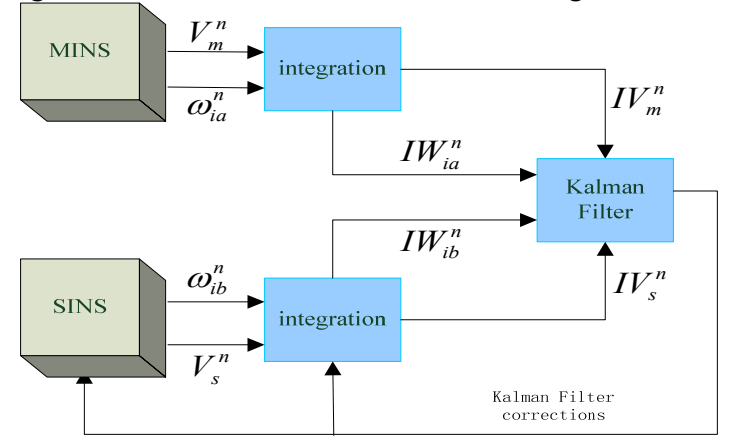

Figure 1. Integrated velocity and integrated angular rate transfer alignment scheme

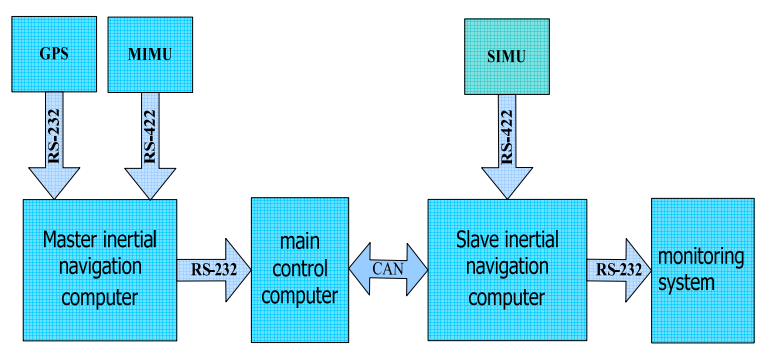

Figure 2. System structure diagram

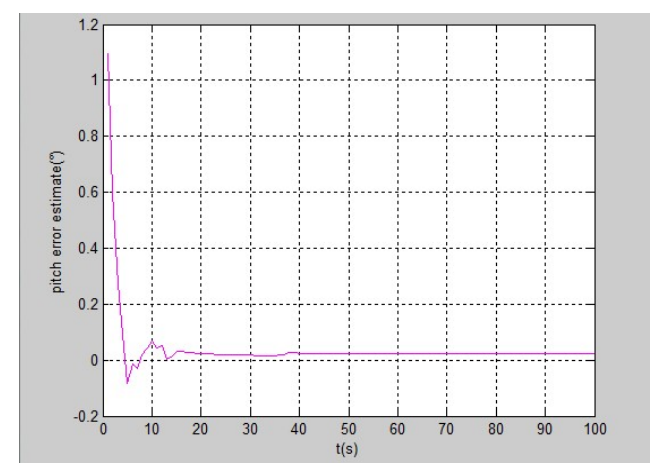

Figure 3. Pitch error estimate 


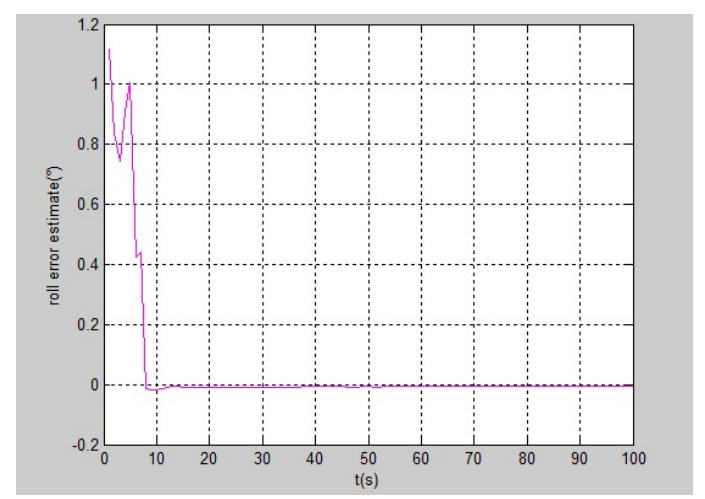

Figure 4. Roll error estimate

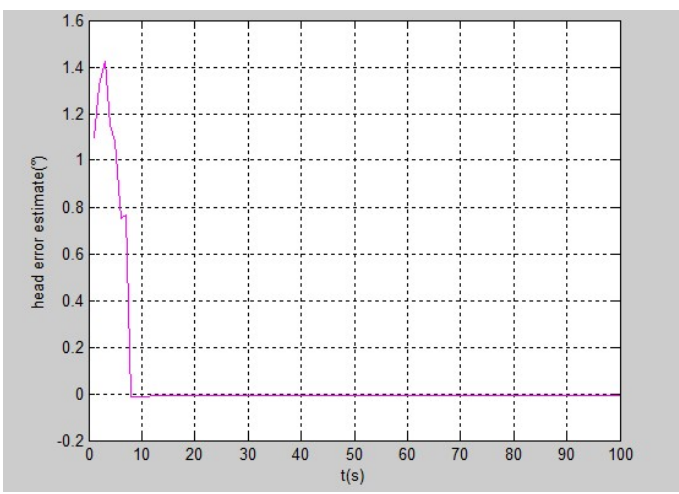

Figure 5. Head error estimate

From Fig.3, it can be seen that pitch error approaches to $0.024^{\circ}$ by " integral velocity and integral angular rate" matching algorithm; From Fig.4, it can be seen that roll error approaches to $0.007^{\circ}$;From Fig.5, it can be seen that head error approaches to $0.008^{\circ}$. The results meet the requirements of distributed inertial attitude measurement system.

\section{CONCLUSION}

To reduce the effect of the deflection to the transfer alignment, we use " integral velocity plus integral angular rate" matching algorithm. The simulation results show that this algorithm has the characteristics of high estimation accuracy, high speed, high reliability and it is able to meet the demand of inertial attitude measurement system for a long time to maintain high accuracy .

\section{ACKNOWLEDGMENT}

The work was supported by NSAF (Grant No U1330133) and the Natural Science Foundation of Jiangsu (Grant BK20130774).

\section{REFERENCES}

[1] D. Jensen, "F-35 integrated sensor suite: Lethal Combination,"Avionics Magazine, 2005(10):22-27.

[2] J. E. Kain and J. R. Cloutier, "Rapid transfer alignment for tactical weapon applications,"AIAA Guidance, Navigation and Control Conference.Boston, MA.pp:1290-1300.AIAA-1989-3581.

[3] R. M. Rogers, "Low dynamic IMU alignment,"Proceedings of the 1998 IEEE Position Location and Navigation Symposium, Apr 20-23 1996. Palm Springs, CA, USA. pp: 272-279.

[4] S. C.Yue, "Research of SINS Transfer Alignment,"Harbin Institute of Technology,2009.(in chinese)

[5] H. S. Guang,Z. H. Yue, "Comparison of Different Transfer Alignment Equations," Journal of Chinese Inertial Technology,2003,11(6):53-63. (in chinese)

[6] M. M. Lin,J. C. Fang and G.J.Gao, "An efficient method of fast and accurate transfer alignment of SINS used on air-to-air missiles ,'Journal of Chinese Inertial Technology,vol.9,no.3,pp:24$28,47,2001$. (in chinese)

[7] A. Gelb.(Ed),Applied optimal estimation, MIT Press, 1974.

[8] K. Spalding, “An efficient rapid transfer alignment filter,"AIAA Paper 92-4598-CP,1992. 